\title{
Germanica
}

\section{Jan Myrdal : l'autobiographie comme apologie}

Autobiographie als Apologie

Elena Balzamo

\section{(2) OpenEdition}

Journals

Édition électronique

URL : http://journals.openedition.org/germanica/1919

DOI : 10.4000/germanica.1919

ISSN : 2107-0784

\section{Éditeur}

Université de Lille

\section{Édition imprimée}

Date de publication : 30 juin 1997

Pagination : 31-45

ISBN : 9782098426320

ISSN : 0984-2632

\section{Référence électronique}

Elena Balzamo, " Jan Myrdal : I'autobiographie comme apologie », Germanica [En ligne], 20 | 1997, mis en ligne le 19 septembre 2013, consulté le 06 octobre 2020. URL : http://journals.openedition.org/ germanica/1919; DOI : https://doi.org/10.4000/germanica.1919

Ce document a été généré automatiquement le 6 octobre 2020.

(ㄷ) Tous droits réservés 


\title{
Jan Myrdal : l'autobiographie comme apologie
}

Autobiographie als Apologie

\author{
Elena Balzamo
}

Dans aucun cas, je ne livre la vérité tout entière Jan Myrdal

1 Jan Myrdal, « enfant terrible » de la littérature suédoise, a beaucoup écrit. Peu de ses œuvres appartiennent à la littérature stricto sensu ; la plupart se meuvent dans l'espace incertain entre le récit de voyage, le reportage, l'enquête sociologique, le journal intime, bref, dans ce domaine paralittéraire qui entoure comme un halo le noyau dur qu'on appelle habituellement la fiction. Cependant, l'auteur refuse catégoriquement l'appellation de journaliste, en revendiquant son droit de faire intervenir des techniques purement littéraires, y compris la fabulation, dans un discours censé donner une image véridique de la réalité objective :

La fiction est la possibilité supérieure pour dépeindre de façon fidèle le cours d'événements sociaux complexes, de manière à ce qu'ils soient intelligibles pour le lecteur - et par conséquent modifiables. La fiction est donc "plus vraie» que n'importe quel récit documentaire ${ }^{1}$.

2 Cette déclaration, le véritable credo de l'auteur, vaut également pour ses livres autobiographiques. Il en a écrit plusieurs : Une enfance en Suède (Barndom, 1982) et Douze ans sur ses treize (Tolv på det trettonde, 1989) racontent respectivement son enfance et son adolescence, tandis que Confessions simultanées d'un intellectuel européen (Samtida bekännelser av en europeisk intellektuell, 1964, paru en français sous le titre de Confessions d'un Européen déloyal) et Confessions d'un Européen déloyal (En illoyal europés bekännelser, 1983, traduction du texte écrit en anglais, Confessions of a Disloyal European, 1968), sont des versions légèrement différentes de sa vie d'adulte.

3 Jan Myrdal refuse d'emblée à l'écriture autobiographique toute spontanéité : non seulement il est persuadé de la nécessité de garder une part de fiction dans un discours 
apparemment non-littéraire, il affirme la nécessité d'appliquer une sévère sélection aux faits du réel qui constitue l'objet de la description autobiographique :

... Je ne révélerai que ce qui me semble indispensable au raisonnement. Toute tentative d'une confession générale [...] aboutit immanquablement à tant de fausseté qu'elle finit par perdre sa valeur. Pour être honnête, je dois préalablement fixer des limites à mon honnêteté. Si les limites sont trop vastes, elles finiront par inclure des zones où je serai inévitablement amené à mentir, et ce mensonge contaminera tout le reste du récit. Je pratique également la censure. Mais je le fais consciemment. Je choisis ce que je considère comme important pour l'objectif que je me suis fixé. Dans aucun cas, je ne livre la vérité tout entière ${ }^{2}$.

Ce passage est révélateur: il contient l'acceptation myrdalienne du pacte référentiel présent dans toute œuvre appartenant à ce genre. Rappelons rapidement le contenu de ce pacte, tel que le formule Ph. Lejeune : «Tous les textes référentiels [= biographies et autobiographies] comportent [...] un pacte référentiel, implicite ou explicite, dans lequel sont inclus une définition du champ du réel visé et un énoncé des modalités et du degré de ressemblance auxquels le texte prétend. $»^{3}$

Dans le passage cité de Myrdal le pacte est donc énoncé : le « champ du réel visé » est délimité et le "degré de ressemblance auquel prétend le texte " est défini ; quant à l'« épreuve de vérification ", l'auteur nous a déjà dit qu'il ne reconnaît que celle qui est conforme à son « objectif ». Or la censure n'est jamais totalement arbitraire, elle se fait toujours au nom de quelque chose. On ne peut faire reposer le droit d'opérer une sélection parmi les éléments de la réalité uniquement sur une reconnaissance de quelque objectif supérieur, d'une vérité qui soit plus haute que celle des faits. Pourtant, l'auteur ne dit pas - ici pas plus qu'ailleurs - au nom de quelle vérité supérieure, ni de quels « objectifs » il s'apprête à ne pas dire « toute la vérité », en s'arrogeant le droit de trier les faits. Dire, comme Myrdal le fait, que la réalité est inépuisable, qu'on ne peut jamais dire « toute » la vérité, est un truisme. Mais lorsque l'auteur déclare ensuite qu'il procédera à une recomposition consciente des faits, cela est déjà plus intéressant, car il s'agit de substituer à la différence quantitative (vérité totale ou partielle) une différence qualitative, en distinguant entre la vérité « utile » (pour « l'objectif que je me suis fixé ») et une vérité inutile, voire même nuisible. Cette distinction entre deux types de vérité restera pertinente pour l'ensemble de textes que nous aborderons ici.

\section{Le mythe du « moi »}

Les deux versions de l'autobiographie adulte de Myrdal, qui se présentent comme des suites de notations chronologiquement disparates, font une part belle aux «flashback » qui renvoient le lecteur à l'enfance de l'auteur-protagoniste. Il convient donc d'examiner d'abord les récits d'enfance mentionnés plus haut pour voir sur quelles bases l'écrivain édifie sa mythologie personnelle.

7 Comme beaucoup de ses compatriotes, Myrdal ne fait pas preuve d'une grande originalité dans le choix du paradigme : on reconnaît aussitôt Le Fils de la servante de Strindberg, le modèle vieux de plus d'un siècle, mais qui continue à exercer une puissante influence sur les écrivains suédois ${ }^{4}$. La conception strindbergienne de l'enfance comme enfer, sa triade fondamentale - «prison de l'enfance », « enfer de la famille ", "martyr de l'école»-se retrouve dans l'œuvre myrdalienne intacte, et même renforcée, si c'est encore possible. Comme le petit Johan chez Strindberg, le petit Jan se sent prisonnier des adultes, étranger à ses parents; comme lui, il souffre le 
martyr à l'école : «Je ne pouvais ni lire ni apprendre quoi que ce fût. L'école volait tout mon temps. $»^{5}$. Mais il y a aussi une différence: si le personnage de Strindberg a intériorisé l'infériorité qui lui avait été imposée, ce n'est pas le cas du héros myrdalien. Malgré sa situation souvent humiliante, il est convaincu de sa supériorité. Jan a six ans lorsqu'il découvre que leur bonne ne sait pas que la terre est ronde :

... j'ai refait cette expérience avec plusieurs adultes, et j'ai trouvé que la plupart ne

se doutaient guère que la terre est ronde ${ }^{6}$.

8 L'infériorité de l'enfant apparaît ainsi comme une injustice, un esclavage dans lequel les adultes le maintiennent et auquel il s'op pose de toutes ses forces. Comme toujours dans ce type de récit, la haine de l'enfant envers son entourage est présentée comme réactive, née du sentiment qu'il est, au sein de sa famille, une victime malaimée et persécutée. Outre son "abandon", il reproche à ses parents de l'avoir considéré comme un enfant à problèmes; il se présente volontiers comme une victime du complot fomenté et entretenu par ses géniteurs qui se répandent en propos calomnieux sur les difficultés qu'ils ont à l'élever.

9 Parmi les mythologèmes du genre (tel qu'il se constitue à partir de Strindberg), on peut citer, outre les rapports antagonistes entre l'enfant et les adultes, le principe d'accumulation des handicaps, héréditaires ou pas, qui tendent à rendre l'enfant inapte à la vie, l'idée de la corruptibilité de l'être naturel qu'est l'enfant, et enfin celle de la haine en tant que moteur et sentiment dominant de son existence. Tous ces traits jouent un grand rôle dans Enfance en Suède et dans Douze ans sur ses treize, deux livres décrivant l'univers cauchemardesque de souffrances et de rage. Plus qu'un règlement des comptes comme il est le plus souvent perçu - il convient de voir dans ces deux textes une élaboration de la mythologie personnelle qui se veut unique, mais qui ressemble à bien d'autres textes et avant tout au Fils de la servante.

10 Ainsi, au fil des pages, se dégage et s'affirme l'image du héros lyrique : d'abord, l'enfant, faible et solitaire, incompris et persécuté, puis l'adolescent qui se cherche, qui se forge, dans un environnement profondément hostile, enfin, l'adulte victorieux, fort et généreux, grand dans ses souffrances (il s'agit le plus souvent des maladies) et immanquablement solitaire. Évoquant l'épisode où le jeune Jan fait un saut périlleux, l'auteur raconte d'abord qu'il était en compagnie de ses camarades, puis se persuade d'avoir été seul au moment de l'exploit : «Je sais qu'un jour j'ai sauté. Je me demande si je ne m'y suis pas rendu seul ce matin de printemps pour voir si j'oserais $»^{7}$. Bien sûr, il ose. Comme dans Enfance en Suède, en évoquant son adolescence et son âge mûr, Myrdal s'arrête volontiers sur les épisodes où il est malade, endure la faim ou le froid, toujours seul, toujours fier, toujours incompris, qu'il souffre des morpions à Oslo, dégringole de sa bicyclette à Stockholm, ou se fracasse le crâne dans la Dalécarlie...

11 Tout héros a besoin d'adversaires. Dans Enfance en Suède et dans Douze ans sur ses treize ce sont avant tout les parents. Dans Confessions d'un Européen déloyal les parents sont pratiquement absents, mais les ennemis ne manquent pas. Sur le chemin de l'école, Jan se bat héroïquement (un contre plusieurs) avec des gamins de la rue et remporte la victoire (morale). Cependant, là aussi, le vrai ennemi, ce n'est pas le voyou ( Je le comprenais. Il me comprenait $\left.»^{8}\right)$, mais un adulte, conseiller-psychologue de son école 
qui ne "comprend pas" les raisons pour lesquelles Jan se bat, pas plus que ses camarades de classe. » Ils ne m'ont pas compris ", ne manque pas de préciser l'auteur9.

Le mythologème central - strindbergien par excellence (et rousseauiste à son origine) est celui du complot; c'est le véritable fil conducteur de l'œuvre de Jan Myrdal. Le monde entier semble s'être ligué contre lui pour lui empoisonner l'existence. On lui gâche le plaisir de jouer, on l'arrache aux États-Unis, le pays qu'il aime, pour le ramener de force en Suède en 1940, on se plaint des difficultés qu'on a à l'élever, on ne comprend pas ses goûts, on ne tient pas compte de ses intérêts etc. :

Depuis la puberté, la musique a été pour moi un besoin. Mais curieusement, durant toute mon enfance et mon adolescence, tous les profs et tous les adultes me répétaient sans cesse que j'étais complètement dépourvu d'oreille musicale... ${ }^{10}$

Plus tard, il n'est plus question de musique, mais des problèmes plus sérieux, et là aussi - le complot :

Ce qu'on voulait de moi - [...] de la part de tous les représentants de la société, des fonctionnaires des partis (sociaux-démocrates et communistes), des "clartéistes ", des éditeurs et des rédacteurs - c'est que je renie moi-même, mes opinions et mes expériences. [...] La vérité supérieure, le bien commun, la tactique du parti exigeaient que je me range ou que j'annonce ma conversion à la Weltanschauung bourgeoise, alors on me pardonnerait beaucoup de choses. Mais je ne pouvais ni l'un ni l'autre ${ }^{11}$.

Selon l'auteur, un livre qu'il a écrit à l'âge de dix-huit ans, un «mélange de peur et de révolte ", pour reprendre ses propres termes, lui aurait valu dix ans de boycott total de tous les éditeurs suédois: "Pendant dix années je n'avais que la plus ample collection

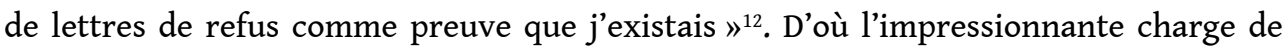
haine qu'il accumule pendant presqu'un quart de siècle et qu'il laisse exploser dans Confessions d'un Européen déloyal :

Il va de soi que je n'ai rien oublié et je n'ai rien pardonné. Je préfère me faire griller en enfer plutôt que tendre le petit doigt pour aider un de ces hommes ou de ces femmes qui, pendant dix ans, m'ont privé de la possibilité de publier un article signé de mon nom. Lorsqu'ils me sourient, je ne leur réponds pas. J'ai désiré leur mort depuis 1946 et je la désire toujours ${ }^{13}$.

15 Persuadé de la gravité des injustices subies, Myrdal refuse de voir une différence entre ses propres déboires avec les maisons d'édition en Suède et les persécutions des intellectuels sous les régimes totalitaires.

A ces complexes enfantins, à ce malaise persistant, à ce sentiment d'être rejeté par tous et partout, l'écrivain n'a qu'une solution, tout aussi puérile : nier la réalité en la fuyant, se réfugier d'abord dans ses rêves, puis à l'étranger où il passera des années d'affilée. Lui-même attribue ce besoin de rompre avec le pays où il ne trouve pas sa place (après avoir rompu avec la famille) à l'incompatibilité entre les exigences de la société et ses dispositions (innées) d'« homme naturel »:

J'ai [...] toujours eu du mal à maîtriser les règles du jeu social. [...] Encore enfant, j'avais remarqué que les autres personnes y étaient bien plus habiles. Jeune, je me disais qu'ils avaient $d u$ « dressage » dans le sang ${ }^{14}$.

17 Cette façon de rejeter la responsabilité de ses échecs (affectifs, sociaux ou professionnels) sur autrui, est bien du Myrdal, mais qui ne fait, là aussi, que suivre le modèle posé par son grand prédécesseur, Strindberg. Dans cet univers-là, le repentir est une notion inconnue. Les Confessions d'un Européen déloyal (sa première version particulièrement) en fournissent un autre exemple éloquent. Ce livre a l'apparence 
d'un auto-plaidoyer (fait sur le modèle évident du Plaidoyer d'un fou), cependant son sens profond est tout autre : alors que Strindberg se défend des fautes qu'il a commises (en expliquant pourquoi il les a faites), Myrdal nous dit en somme ceci : regardez comme j'ai la conscience vive et délicate : je suis prêt à endosser la responsabilité d'un crime dont je ne suis pas coupable (il s'agit d'un suicide qu'il avait pressenti, mais qu'il n'a pas empêché). Or, c'est faux : il n'endosse pas ce crime, mais s'en décharge sur sa condition. A la fin du livre, le lecteur apprend que Jan Myrdal, brave et généreux en tant qu'individu - ne prête-t-il pas son appartement (il en a un autre) à une pauvre jeune fille, après avoir héroïquement vaincu son désir de coucher avec elle?-, ce Jan Myrdal en tant qu'intellectuel européen, produit de la société capitaliste, s'avoue coupable de l'indifférence qui est la cause indirecte du suicide de cette jeune fille !

« Comme j'ai trahi A., ainsi nous avons toujours trahi. [...] Nous qui nous inscrivons

dans une tradition - européenne - [...] nous avons trahi en connaissance de cause.

Nous avons analysé toutes les guerres avant qu'elles eussent été déclarées. Mais

nous ne les avons pas empêchées ${ }^{15}$ etc., etc.

Le procès que Myrdal fait à l'intelligentsia est très bref, un seul paragraphe, quatorze lignes et demie (pour en mesurer la légèreté, pensons ne serait-ce qu'à la Trahison des clercs de Julien Benda). Il relève d'un véritable tour de force, car même si le mot « intellectuel » figure dans le titre du livre, rien dans le texte n'autorise l'identification. Pendant cent cinquante pages Myrdal explique qu'il n'est pas «comme les autres", pour annoncer à la page 151 que s'il a commis des fautes, c'est parce qu'il fait partie de l'intelligentsia européenne. Bien entendu, c'est l'unique fois qu'il emploie le pronom personnel «nous » au pluriel. (Cf. Douze ans sur ses treize où tout le texte est habilement construit autour de l'opposition «moi » / « eux »). Pour Jan Myrdal, il n'existe pas de «nous », seul le « moi » existe, et même ce « moi », il a du mal à le définir.

La quête de soi aboutit à une impasse : en s'arrogeant le droit de trier les faits, de les censurer à sa guise, bref, de mentir, l'auteur se révèle incapable de les maîtriser. La vérité échappe, elle suinte ici et là : un aveu involontaire, un lapsus mal contrôlé, un détail révélateur, et voilà que derrière un Zorro fort et sûr de lui, derrière un chevalier sans peur et sans reproche - pourquoi pas un Don Quichotte combattant le mal aux quatre coins du monde? - apparaît un enfant timide, complexé, persuadé que tout le monde n'a de regards que pour lui, qu'on lui en veut, qu'il est seul, entouré d'adultes dangereux et méchants. On retrouvera ces fantasmes, constitutifs des écrits autobiographiques de Myrdal, intacts dans ses ouvrages politiques.

\section{Le mythe de « l'autre »}

Toute mythologie personnelle comporte deux volets: à côté de l'image du «moi", l'auteur crée - plus ou moins consciemment - l'image du monde dans lequel ce «moi » évolue. En tant que créateur de la première, l'auteur de l'autobiographie est avant tout un analyste; en tant que créateur de la seconde il est surtout un témoin. Dans l'œuvre de Myrdal, ce double rôle de l'écrivain est patent: quand il n'écrit pas sur lui-même, il écrit sur la "réalité sociale». Mais avant de voir si un homme ayant une mythologie personnelle aussi encombrante peut s'acquitter convenablement du rôle de témoin, il convient de s'arrêter sur les techniques qu'il emploie pour décrire le monde extérieur dans ses récits strictement autobiographiques. 
21 Il s'agit immanquablement d'un espace hostile, peuplé d'ennemis. Pourtant, malgré la quantité impressionnante de ces derniers, on a souvent l'impression que l'existence du personnage central se déroule dans le vide. Outre le fait que l'intérêt de l'auteur est entièrement absorbé par sa propre personne, au point que sa femme n'occupe pas plus de place dans sa narration que son chien ou sa collection des disques, on y relève une technique permettant de plonger les gens qui l'entourent dans un anonymat quasi total : ils sont tous désignés par leurs initiales. Les personnages défilent sans nom ni visage (leur physique n'est jamais décrit, tandis que l'auteur ne perd jamais l'occasion de mentionner sa propre tenue, d'informer le lecteur s'il a le teint bronzé, s'il a maigri etc.). Les êtres sont tirés du néant, utilisés dans une mise en scène quelconque, puis rejetés là d'où ils sont venus. En fait, le monde entier peut être effacé de la sorte - il suffit de fermer les yeux. Face à une situation désagréable, explique Myrdal, on n'avait qu'à se persuader que cette situation n'existait pas. En regardant fixement les gens qui l'entouraient, le petit Jan avait le sentiment qu'ils devenaient de plus en plus flous, transparents, avant de disparaître tout à fait. Dès cet instant, le champ libre était donné aux rêveries, aux « rêves diurnes » :

Comme la plupart des enfants [...] j'avais trouvé et perfectionné un système magique pour maitriser le monde [...] si discret que les adultes ne soupçonnaient rien et ne pouvaient intervenir. [...] Il suffisait de plisser les yeux pour que les adultes déplaisants - les professeurs, les agents de police - devinssent flous et disparaissent dans un brouillard... ${ }^{16}$

L'anéantissement imaginaire de la réalité qui fait face est paradigmatique de la totalité de l'œuvre myrdalienne. Voyons maintenant en détail comment ce mécanisme fonctionne dans les textes où le rôle de l'auteur est celui de témoin.

Nous avons choisi comme échantillon des "témoignages" de Myrdal une série d'articles sur l'Albanie qu'il a visitée pour le compte de l'Expressen en 1986 (parus entre le 8 et le 12 novembre). Ces textes sont relativement innocents par comparaison avec les monstruosités qu'il a écrites sur la chine, ou avec son premier livre consacré à l'Albanie, publié en 1970 sous le titre Défi albanais (Albansk utmaning), mais ils suffisent pour démontrer les méthodes de sélection et de montage qu'il utilise.

24 L'Albanie était alors un des pays les plus fermés du monde ; les Occidentaux autorisés à s'y rendre étaient rares et leur témoignage pesait d'autant plus lourd. En tant qu'Intellectuel Européen, Jan Myrdal ne pouvait l'ignorer. On ne peut donc attribuer ses déclarations à l'effet de surprise : Myrdal visitant l'Albanie en 1986 n'est pas Gide se rendant en Russie au début des années 30 ; notre auteur avait à plusieurs reprises séjourné dans des pays communistes, y compris en Albanie, en 1968. L'effet troublant de ses «témoignages » vient du fait que le genre de reportage obéit, chez Myrdal, aux mêmes mécanismes que ses écrits autobiographiques: mêmes fantasmes, même répartition des rôles, même glissement de la vérité vers le mensonge, diffus et omniprésent.

L'Albanie apparaît dans cette série d'articles comme un alter ego de l'auteur : c'est un pays-enfant (dans le premier article de la série, l'adjectif « petit » revient six fois, tout autant que le mot "grand» pour qualifier les autres États). Un pays qui vit dans un environnement hostile, obligé de se défendre face aux innombrables ennemis : victime tantôt de "menaces militaires ", tantôt d'une "guerre économique (subversive!)», menacé par l'« alliance tactique de la diplomatie des grandes puissances ». 
Ce «petit Poucet » qui, selon l'auteur, « dans l'Europe de 1986 représente le grand défi politique et social», est ontologiquement différent des autres pays (aussi bien capitalistes que communistes) tout comme Jan Myrdal-enfant est ontologiquement différent non seulement des autres enfants (de ses sœurs, par exemple) mais de tous les adultes. La spécificité de l'Albanie consiste en ce que c'est «l'unique pays de l'Europe de l'Est où le peuple est solidaire de ses dirigeants ». Quelle est la cause de ce miracle ? Par la bouche d'un de ses interlocuteurs, Jan Myrdal nous la révèle : « dans sa défense de la souveraineté nationale, le régime a le soutien quasi unanime de la population ».

Apparemment, c'est à son amour de la liberté que l'Albanie doit ses déboires économiques : «même la génération d'aujourd'hui paie cher l'indépendance », car «les deux libertés, celle de la nation et celle du marché économique sont inconciliables ", déclare-t-il, avant d'ajouter :

Si on comprend les droits de l'homme à la manière du Conseil européen, comme en France en 1789, et qu'on y fasse figurer le droit inaliénable à la propriété privée, alors ces mesures [construction du socialisme en Albanie] sont incompatibles avec ce que chez nous on appelle solennellement les Droits de l'homme.

Ses succès économiques, le pays les doit «aux gigantesques injections rigoureusement planifiées par l'État - des capitaux et à la main-d'œuvre bénévole ». Lorsque le prix du café monte sur le marché mondial, en Albanie «il reste stable car là règne la dictature prolétarienne et la politique économique de Staline ». Voilà pour les succès, mais quand il s'agit de revers, la faute en est toujours aux autres, aux « adultes » (Russes, Chinois, Occidentaux) : "Voilà qu'à présent la crise économique mondiale met l'économie du pays devant de nouveaux problèmes graves ». Le monde entier, depuis les pays producteurs d'huile d'olive jusqu'aux exportateurs du café, sont ligués contre la « petite » Albanie.

Myrdal se rend-il compte combien peu originale est cette façon de voir les choses ? Sait-il qu'on a inculqué à des générations d'est-européens cette mentalité de «forteresse assiégée » qui, d'ailleurs, n'a rien à voir avec la taille du pays : les Russes eux-mêmes la partagent avec les Chinois? Pour lui, la haine qui domine les rapports entre le petit Jan et ses parents, régit également les rapports entre les nations. Comme l'enfant (Jan Myrdal) est naturellement bon et que toutes ses actions sont des réactions aux agressions extérieures, ainsi l'Albanie ne fait que répondre au défi par ses choix politiques et économiques, par son autarcie et par la terreur (idéologique, politique et culturelle) que les autorités font régner sur le « territoire national».

Parmi les « systèmes magiques pour maîtriser le monde » élaborés par Myrdal-enfant, il y en avait un qui consistait à imaginer qu'on construit un mur entre soi-même et son entourage ; le mur achevé, on est à l'abri : le monde extérieur n'a plus de prise sur vous. C'est exactement le comportement qu'il prête à l'Albanie. Ce nouveau David résiste victorieusement aux assauts des ennemis: tout comme Myrdal-enfant réussit à préserver son intégrité, l'Albanie sauve son indépendance au prix de l'isolement total, d'une fermeture hermétique au monde extérieur : «Pauvre, mais indépendant », ainsi résume la situation du pays un apparatchik porte-parole de l'auteur, comme si l'un excluait vraiment l'autre. "Pauvre, continue ce dialecticien, mais on supporte mieux l'égalité et la pauvreté quand elles sont partagées ».

31 On penserait cependant que ces élucubrations d'une imagination échauffée devraient voler en éclats au premier contact avec la réalité. Nullement : non seulement la réalité que Myrdal voit n'apporte aucun correctif à ses opinions, mais elle les renforce. C'est là 
un des phénomènes les plus terrifiants du fonctionnement mental des pseudointellectuels du xxe siècle, mécanisme qui fait que les uns doutent de l'existence du Goulag, et que les autres ne reconnaissent pas les chambres à gaz même lorsqu'on les amène devant elles.

Jan Myrdal se présente volontiers comme témoin. Or, c'est précisément ce qu'il ne peut pas être, car il ne voit que ce qu'il a décidé de voir. Une des photos qui accompagnent ses reportages montre un Albanais à côté de sa moto. Myrdal commente :

Il existe, bien entendu, des passionnés de la moto même en Albanie. A Gjirokastra nous rencontrâmes Pavli Daka, photographe et amateur de motos [...]. Il a amoureusement soigné et réparé une Banelli italienne de 1935 qui brille à présent comme une neuve.

$\mathrm{Au}$ premier abord, l'auteur fait preuve d'une exactitude et d'une véracité exemplaires : il n'a omis ni le nom de la personne, ni son métier, ni l'endroit où il l'a rencontré, ni la marque de la moto, ni l'année. Il a seulement « oublié » de communiquer à ses lecteurs ce détail : si en Occident le fait d'être propriétaire d'une voiture ou d'une moto des années 30 est un indice de niveau social élevé et d'un choix délibéré du véhicule, dans l'univers socialiste, c'est précisément le contraire : un signe de pauvreté, de l'absence des moyens et du choix, seuls les membres de la nomenklatura pouvant se permettre d'avoir une moto ou une auto d'une marque récente. Ce détail anecdotique prépare, un peu plus loin, un mensonge bien plus gros : si à Tirana tout le monde se déplace à pied ou à bicyclette, déclare Jan Myrdal, c'est uniquement par souci de préserver l'environnement!

Dès lors, le lecteur ne s'étonne plus en apprenant qu'en Albanie les problèmes économiques sont résolus grâce au "travail physique volontairement choisi par la jeunesse du pays (entre 14 et 22 ans) et à la planification scientifique ». Ce travail " volontaire " ne serait pas plus obligatoire que l'école, « mais tous les enfants y vont ". Sans doute pour accroître la liberté de cette jeunesse heureuse,

chez nous, on n'a pas le droit de changer de domicile, [dit un dignitaire du parti à la retraite, type préféré d'interlocuteurs de Myrdal - E.B.]. Nous n'acceptons pas la liberté qui est nuisible. [...] Beaucoup de jeunes ne sont pas contents de cela [...], mais ça ne dure que cinq ou six ans, jusqu'à ce qu'ils aient fondé une famille. Alors ils comprennent que le parti avait raison et qu'il a agi pour leur propre bien ainsi que pour le bien du pays, et c'est ce qu'à leur tour ils diront à leurs jeunes frères et sœurs, et plus tard à leurs propres enfants. Le parti sait prendre ses responsabilités.

Le rêve totalitaire fait ici bon ménage avec l'anarchisme. L'auteur ne semble nullement gêné par la contradiction entre ses déclarations telles que "c'est à l'idée et non pas à l'idée organisée qu'on doit sa fidélité » et le fait que le parti communiste albanais n'est autre chose que ce qu'il appelle maladroitement l'« idée organisée ».

Le besoin de conformer la réalité à ses désirs amène Myrdal à aligner les contradictions les plus aberrantes: tantôt il explique que «la véritable libération des femmes passe par l'économie planifiée» (qui subventionne l'industrie textile pour permettre aux femmes d'acheter des robes au lieu de se confectionner des vêtements traditionnels), tantôt il fait écho aux autorités affirmant que si on a fermé l'accès aux produits culturels de l'Occident c'est uniquement pour préserver la culture albanaise traditionnelle. La démarche est caractéristique: le refus de toute forme de collectivisme tant qu'il est question de soi-même, va de pair avec l'éloge du collectivisme et des valeurs collectives lorsqu'il est question des autres. 

néfastes.
Désormais on comprend mieux le mécanisme qui pousse Myrdal à chercher à rétablir la justice dans des pays exotiques (Inde, Amérique latine etc.) où il se donne le beau rôle de héros solitaire, rôle d'autant plus facile qu'il y va non pas pour faire quelque chose de concret, mais pour se donner les moyens d'en parler, chez lui, en Europe : audacieux, mais point téméraire. Cela lui offre également une excuse : l'engagement " ailleurs » le dispense du devoir d'engagement chez lui, en Suède (ou en Europe). Il s'engage pour mieux se désengager.

Celui qui se veut témoin impartial devient un jouet docile aux mains du régime qui lui est cher (l'interprétation freudienne est ici trop évidente et donc sans intérêt); la recherche de la vérité prend la forme d'un opportunisme de la pire espèce, d'un aveuglement volontaire dans les pures traditions des vieux communistes qui acceptaient de se calomnier eux-mêmes (ainsi que leurs amis) si le parti l'exigeait (en 1964 Jan Myrdal en est d'ailleurs toujours resté au stade des interrogations puériles : « La question du rôle qu'a joué Radek et de la conscience de Radek continue à être pour moi un grand problème $\aleph^{17}$ ).

Son individualisme forcené (lié à son immaturité) se retourne en son contraire et devient un renoncement volontaire au droit de voir par ses propres yeux et de juger par lui-même. Il va en Albanie en tant que privilégié (il omet d'expliquer pourquoi le régime, qui cherche à minimiser ses contacts avec le monde extérieur, ouvre ses portes à Jan Myrdal, citoyen suédois et «intellectuel européen ») et il se fait le porte-parole des apparatchiks albanais tout en croyant dire ses quatre vérités au monde entier. Les milliers d'Albanais qui se rueront sur l'Italie et la Grèce un peu plus tard, se faisant mitrailler par les gardes frontières, font partie des faits qu'on a le « droit d'ignorer », ils se trouvent probablement en dehors des «limites d'honnêteté » que s'est fixées l'auteur. Sans se douter de rien, il continue à répéter les sornettes officielles sur l'« unité profonde entre le peuple et le régime ».

«La vérité politique est concrète », annonce Myrdal dans un de ses articles. En croyant faire preuve d'une audace intellectuelle, il ne fait, une fois de plus, que confirmer son opportunisme profond. Enfant révolté, tremblant de haine et de mépris, il se gardait bien de les laisser transparaître : «Je me taisais, le sourire aux lèvres ", c'est le refrain de Douze ans sur ses treize. Si en 1986 il avait quelques réserves sur le régime albanais, il n'en laissa rien transparaître.

$\mathrm{Au}$ début des Confessions d'un Européen déloyal, l'auteur pré vient qu'il donne au mot « bekännelse » (confession) un sens peu orthodoxe :

Même si je considère mon occupation présente comme un «examen de conscience », je vois dans la 'confession' davantage une apologie qu'un aveu ${ }^{18}$.

2 Le lecteur se trouve, en effet, devant une auto-apologie déroutante, dont la ferveur fait penser à la fuite en avant d'un enfant entassant des mensonges de peur d'être interrompu et rappelé à l'ordre.

3 Chez Jan Myrdal, l'élaboration du mythe personnel, inhérente à toute écriture autobiographique, a, comme condition indispensable, la négation systématique du monde extérieur. La démolition acharnée du réel, à laquelle il se livre dans la plupart de ses écrits, ne peut manquer de déteindre sur le personnage qu'il peint. Son œuvre offre un cas édifiant d'une quête de la vérité qui se solde par un mensonge des plus 


\section{NOTES}

1. - Cité par M. Fors et L. Andrée dans "Jan Myrdal, ou le voyage des livres" dans Europe. Littérature Suédoise, avril 1994, p. 82.

2. - J. Myrdal, Samtida bekännelser av en europeisk intellektuel, Sthlm 1964, pp. 15-6.

3. - Ph. Lejeune, Le pacte autobiographique, Seuil 1975, p. 36.

4. - Cf. notre article "Récit d'enfance : mythe ou réalité ? Esquisse d'une typologie littéraire » dans : Germanica, XV, 1994, et notamment le passage consacré à Enfance en Suède de J. Myrdal, pp. 181-185.

5. - Ibid., p. 22.

6. - J. Myrdal, En illojal europés bekännelser, Norstedts, Sthlm 1983, p. 70.

7. - Ibid., p. 40.

8. - J. Myrdal, Tolv på det trettonde, Sthlm 1989, p. 40.

9. - Ibid., pp. 39-40.

10. - Ibid., p. 72.

11. - Ibid., pp. 128-129.

12. - J. Myrdal, En illojal europés bekännelser, p. 220.

13. - Ibid., p. 221.

14. - J. Myrdal, Tolv på det trettonde, p. 17.

15. - Ibid., p. 151.

16. - J. Myrdal, En illojal europés bekännelser, pp. 33-34.

17. - Ibid., p. 109.

18. - J. Myrdal, En illojal europés bekännelser, p. 10.

\section{RÉSUMÉS}

Le premier volet de l'article présente une analyse des ouvrages autobiographiques de Jan Myrdal, écrivain suédois contemporain, en s'efforçant, d'une part, de décrire les mécanismes opérant lors de l'élaboration d'une "mythologie personnelle», et, d'autre part, d'indiquer le rôle que jouent dans cette élaboration les modèles littéraires préexistants (et notamment les écrits autobiographiques de Strindberg). Le second volet cherche à démontrer comment, une fois mise en place, cette mythologie personnelle détermine à son tour la vision de la "réalité sociale " quand celle-ci devient l'objet d'une description. Le lien intime entre le «mythe de soi » et le "mythe de l'autre » est ainsi mis en évidence. L'intérêt des écrits autobiographiques de Myrdal réside dans le fait que, tout en constituant un cas extrême de ce que nous appelons « la négation du réel », il ne reste pas moins le représentant typique d'une importante famille d'intellectuels.

Der erste Teil des Artikels analysiert die autobiographischen Werke des zeitgenossischen schwedischen Autors Jan Myrdal unter zwei verschiedenen Gesichtspunkten. Einerseits werden die bei der Herausbildung einer persönlichen Mythologie wirkenden Mechanismen beschrieben, andererseits die Rolle der ursprünglichen literarischen Modelle (inbesondere die autobiographischen Schriften Strindbergs) bei diesem Prozeß herausgearbeitet. Der zweite Teil soll zeigen, wie diese persönliche Mythologie ihrerseits die Vorstellung von der sozialen Realität 
beeinflußt, wenn diese zum Gegenstand der Beschreibung wird. Auf diese Weise wird der enge Zusammenhang zwischen dem «Mythos des Eigenen» und dem «Mythos des Anderen» offengelegt. Die Tatsache, daß Myrdal einen Extremfall dessen darstellt, was wir «Negation des Reellen» nennen, kann das Interesse an seinen autobiographischen Schriften nicht trüben, ist er doch ein Typischer Vertreter einer wichtigen intellektuellen Familie. 